\title{
Gene interactions constrain the course of evolution of phosphine resistance in the lesser grain borer, Rhyzopertha dominica
}

\author{
DI Schlipalius ${ }^{1,2}$, W Chen ${ }^{3}$, PJ Collins ${ }^{2,4}$, T Nguyen ${ }^{3,5}$, PEB Reilly ${ }^{3}$ and PR Ebert ${ }^{1,3}$ \\ ${ }^{1}$ School of Integrative Biology, University of Queensland, St Lucia, Queensland, Australia; ${ }^{2}$ Cooperative Research Centre for National \\ Plant Biosecurity, Canberra, Australian Capital Territory, Australia; ${ }^{3}$ School of Molecular and Microbial Sciences, University of \\ Queensland, St Lucia, Queensland, Australia and ${ }^{4} Q u e e n s l a n d$ Department of Primary Industries and Fisheries, Indooroopilly, \\ Queensland, Australia
}

\begin{abstract}
Phosphine, a widely used fumigant for the protection of stored grain from insect pests, kills organisms indirectly by inducing oxidative stress. High levels of heritable resistance to phosphine in the insect pest of stored grain, Rhyzopertha dominica have been detected in Asia, Australia and South America. In order to understand the evolution of phosphine resistance and to isolate the responsible genes, we have undertaken genetic linkage analysis of fully sensitive (QRD14), moderately resistant (QRD369) and highly resistant (QRD569) strains of $R$. dominica collected in Australia. We previously determined that two loci, rph1 and rph2, confer high-level resistance on strain QRD569, which was collected in 1997. We have now confirmed that rph1 is responsible for the moderate resistance of strain QRD369, which was collected in 1990, and is shared with a
\end{abstract}

highly resistant strain from the same geographical region, QRD569. In contrast, rph2 by itself confers only very weak resistance, either as a heterozygote or as a homozygote and was not discovered in the field until weak resistance (probably due to rph1) had become ubiquitous. Thus, high-level resistance against phosphine has evolved via stepwise acquisition of resistance alleles, first at rph1 and thereafter at rph2. The semi-dominance of rph2 together with the synergistic interaction between rph1 and rph2 would have led to rapid selection for homozygosity. A lack of visible fitness cost associated with alleles at either locus suggests that the resistance phenotype will persist in the field.

Heredity (2008) 100, 506-516; doi:10.1038/hdy.2008.4; published online 13 February 2008

Keywords: pesticide resistance; fitness; methyl bromide; oxidative stress; linkage mapping; RAF

\section{Introduction}

The primary means of controlling insect pests in stored grain is by fumigation with phosphine, but genetic resistance towards this fumigant has emerged across the globe. Very high levels of resistance have now been reported in pest insects from Bangladesh (Mills, 1983; Tyler et al., 1983), India (Rajendran and Narasimhan, 1994b), China (Ren et al., 1994), Brazil (Lorini et al., 2007) and Australia (Collins, 1998). Species with high-level resistance reported include Rhyzopertha dominica (Collins, 1998; Lorini et al., 2007), Tribolium castaneum (Collins, 1998), Sitophilus oryzae (Nayak et al., 2003; Benhalima et al., 2004), Lasioderma serricorne (Rajendran and Narasimhan, 1994a), Liposcelis decolor and L. entomophilia (Nayak et al., 2003). With the global ban on the use of methyl bromide and the resulting increase in the use of phosphine, high levels of phosphine resistance in insect pests is set to become a serious problem world-

Correspondence: Dr D Schlipalius, School of Integrative Biology, University of Queensland, St Lucia, Queensland 4072, Australia.

E-mail: david.schlipalius@uq.edu.au

${ }^{5}$ Current address: Biotechnology Centre, Nong Lam University, Ho Chi Minh City, Vietnam.

Received 11 January 2007; revised 18 December 2007; accepted 5 January 2008; published online 13 February 2008 wide. Because there are no suitable replacements for phosphine, it is important to understand the genetic factors that have contributed to resistance so that selection for even higher resistance levels can be avoided or delayed. Also, since phosphine appears to induce oxidative stress via the generation of oxyradicals (Bolter and Chefurka, 1990; Chaudhry and Price, 1992), the genes responsible for phosphine resistance may also be responsible for an adaptive defence response against oxidative stress.

The lesser grain borer, $R$. dominica is a destructive beetle that bores into intact grain kernels of most cereals and is considered a major pest of stored grain worldwide. In this species, moderate-level phosphine resistance in Australia was first reported as being widespread across the eastern part of the country in 1990 (White and Lambkin, 1990). It is against this backdrop that high-level resistance emerged in 1997 (Collins et al., 2002). A strain exhibiting moderate level resistance (strain QRD369) fortuitously had been collected in 1990 from the same geographical region in which high-level resistance eventually emerged (strain QRD569), the highly resistant strain used in this study.

In a previous study we showed that the majority of high-level phosphine resistance in QRD569 is caused by two major resistance loci (Schlipalius et al., 2002), 
subsequently named rph1 and rph2 (Schlipalius, 2004; Schlipalius et al., 2006). Using the markers for these resistance loci (named rp6.79 and rp5.11 for rph1 and $r p h 2$, respectively), we were able to show that when present in a homozygous state these two resistance genes were shown to separately convey a weak resistance phenotype $(\sim 50 \times$ and $\sim 12.5 \times$ respectively). However, when both were homozygous in the same individual, they act synergistically to produce a resistance phenotype far greater than the sum of their individual contributions $(>250 \times)$. Classical genetic analysis of strain QRD369 suggested that it had a major resistance gene plus one or more minor factors and that the primary resistance factor in QRD369 was also present in QRD569 (Collins et al., 2002). The identity of the major gene in QRD369 was unknown and the interactions between the two genes in strain QRD569 had likewise not been explored.

Moreover, using genetic crosses to two different susceptible strains (QRD14 and QRD63) the relative fitness of weak phosphine-resistant $R$. dominica (QRD369) was assessed using a caged population approach and phenotype assay (Collins et al., 2001). It was concluded that there was no significant difference in fitness between resistant and susceptible phenotypes for QRD369 over five generations.

In this study we provide a continuation of the previous work (Schlipalius et al., 2002) and use molecular markers to further refine the chromosomal positions of the rph1 and $r p h 2$ resistance loci, describe the genetic interactions between resistance loci and describe the relative fitness of the rph 2 resistance locus in the absence of phosphine exposure. Knowledge of the factors that contribute to resistance will facilitate the proper management of resistance as it currently exists, providing data that will inform decision-making and facilitate modelling of the spread of resistance.

\section{Materials and methods}

\section{Fumigations}

Fumigation of insects was performed in sealed airtight chambers at $25^{\circ} \mathrm{C}$ for $48 \mathrm{~h}$, with recovery on whole grain at $25^{\circ} \mathrm{C}$ for 14 days as described by Daglish and Collins (1998).

\section{Beetle strains and genetic crosses}

The three strains used in this study are described by Collins et al. (2002). The phosphine-susceptible strain (QRD14) was derived from adults collected from a central storage facility in Oakey, southeast Queensland in 1971. The moderately resistant strain (QRD369) was derived from adults collected from on-farm storage at Condamine, southeast Queensland in 1990. The highly resistant strain (QRD569) was collected from a phosphine control failure at a central storage in Millmerran in southeast Queensland in 1997. These three locations exist within a 50-km radius. All strains were cultured in whole wheat at $30{ }^{\circ} \mathrm{C}$ and $55 \%$ relative humidity.

\section{Intercross between moderately resistant and sensitive strains}

A sensitive (QRD14) virgin female was mated with a resistant (QRD369) male to produce $\mathrm{F}_{1}$ hybrids. A single virgin $F_{1}$ female was then crossed with a sibling male to produce an $F_{2}$ population. The $F_{2}$ generation was allowed to mate freely to produce an $\mathrm{F}_{3}$ population. After a limited mating period, 42 individuals of the $F_{2}$ population were removed to be used in linkage analysis. The bulk $\mathrm{F}_{3}$ was used to produce an $\mathrm{F}_{4}$ population. A total of $400 \mathrm{~F}_{4}$ beetles were then treated with phosphine with a dose that kills all susceptible beetles and leaves only resistant survivors, that is a discriminating dose $\left(0.04 \mathrm{mg} \mathrm{l}^{-1}\right.$ for $\left.48 \mathrm{~h}\right)$, and allowed to recover for 14 days at $25^{\circ} \mathrm{C}$. Eighty survivors from this dose were used to find markers tightly linked to phosphine resistance via DNA fingerprinting. Subsequent generations were produced by random mating of unselected beetles. A detailed response curve was created using $\mathrm{F}_{8}$ beetles. These individuals were fumigated with phosphine at $0.007,0.01,0.015,0.02$ and $0.05 \mathrm{mg} \mathrm{l}^{-1}$ and $0.07 \mathrm{mg} \mathrm{l}^{-1}$ for $48 \mathrm{~h}$

\section{Backcross to the moderately resistant parent}

An $F_{1}$ female (sibling to the $F_{1}$ used in the intercross) was backcrossed to the resistant male parent to produce a backcross population of 91 individuals. These beetles (46 and 45 individuals respectively) were subsequently selected at two doses, 0.015 and $0.04 \mathrm{mg} \mathrm{l}^{-1}$ phosphine, both of which produce an lethal dose (LD) 50 for the backcross strain. Two doses were used to confirm the $\mathrm{LD}_{50}$ plateau response of the backcross (Collins et al., 2002).

\section{Strong resistance intercross}

The intercross population segregating for high-level resistance was performed as described previously (Schlipalius et al., 2002). A sensitive (QRD14) virgin female was mated with a highly resistant (QRD569) male to produce $F_{1}$ hybrids. A single virgin $F_{1}$ female was then crossed with a sibling male to produce an $\mathrm{F}_{2}$ population of 104 individuals from which 92 were chosen as a mapping population. This mapping population was not exposed to phosphine, but rather was used to produce a framework genetic map with which to determine the genomic location of DNA markers linked to the resistance trait in the line QRD569. Virgin sibling $\mathrm{F}_{2}$ beetles were collected for the mapping population and the premature death of the $F_{1}$ female left only twelve sibling $\mathrm{F}_{2}$ individuals with which to initiate an $\mathrm{F}_{3}$ population. Untreated beetles were selected from this line at the $F_{5}, F_{10}$ and $F_{20}$ generations for fitness analysis.

\section{DNA fingerprinting}

DNA extraction methods, PCR conditions and electrophoretic separation were as previously described (Schlipalius et al., 2001). Beetles were snap frozen in liquid nitrogen and DNA was extracted from individuals by homogenization with a pestle in a microcentrifuge tube followed by boiling in $1 \mathrm{ml}$ of a 5\% Chelex-100 (Bio-Rad, Sydney, New South Wales, Australia) suspension in TE buffer (100 mM Tris- $\mathrm{HCl}, \mathrm{pH} 8.0,1 \mathrm{mM}$ EDTA, $2.5 \times 10^{-7} \mathrm{mgl}^{-1}$ RNase A) for $15 \mathrm{~min}$. DNA markers were generated using arbitrary 10 or 11 mer primers by random amplified DNA fingerprinting (RAF) analysis performed on individuals. Each reaction volume of $10 \mu \mathrm{l}$ contained $1 \mu \mathrm{l} 10 \times$ PCR buffer $(100 \mathrm{mM}$ Tris-HCl, pH 8.0, $100 \mathrm{mM} \mathrm{KCl}, 50 \mathrm{mM} \mathrm{MgCl}_{2}$ ), $0.2 \mu \mathrm{l} 1 \mathrm{mM}$ deoxyribonu- 
cleotide triphosphates (dNTPs), $0.15 \mu 110 \mathrm{U}^{-1} \mathrm{l}^{-1}$ AmpliTaq Stoffel Fragment DNA polymerase (Perkin Elmer, Melbourne, Victoria, Australia), $2.5 \mu \mathrm{Ci}$ per $10 \mu \mathrm{l}$ $\alpha$-labelled ${ }^{33} \mathrm{P}$-dATP, $5 \mu \mathrm{l}$ of $10 \mu \mathrm{M}$ primer and $1 \mu \mathrm{l}$ of 1:10 diluted template DNA stock in a total volume of $10 \mu \mathrm{l}$. The PCR reaction was pre-incubated at $94{ }^{\circ} \mathrm{C}$ for $5 \mathrm{~min}$; then subjected to 30 cycles of $94^{\circ} \mathrm{C}$ for $30 \mathrm{~s}, 57^{\circ} \mathrm{C}$ for $1 \mathrm{~min}, 56^{\circ} \mathrm{C}$ for $1 \mathrm{~min}, 55^{\circ} \mathrm{C}$ for $1 \mathrm{~min}, 54^{\circ} \mathrm{C}$ for $1 \mathrm{~min}$, $53{ }^{\circ} \mathrm{C}$ for $1 \mathrm{~min}$; with a final extension of $72{ }^{\circ} \mathrm{C}$ for $5 \mathrm{~min}$. Resulting radiolabelled PCR products were separated on $4 \%$ polyacrylamide manual sequencing gels and visualized by overnight exposure of Biomax MR (Kodak) autoradiography film to the dried gels. The polyacrylamide gel separation conditions were as consistent as possible between all DNA fingerprinting analyses (including the previously published analyses), to facilitate comparison.

\section{Resistance gene mapping in the moderately resistant strain}

For linkage analysis of the intercross, DNA fingerprints from the resistant and sensitive parents, as well as from the $F_{1}$ hybrids, were compared with the fingerprints of 42 unselected $\mathrm{F}_{2}$ intercross progeny. Similarly, linkage analysis of the backcross used DNA fingerprints from the resistant and sensitive parents, the $F_{1}$ hybrid and the 91 backcross progeny. Analysis of genetic linkage of markers was performed using Map Manager QTXb06 software for the Macintosh (Manly and Olson, 1999). In cases where a PCR reaction failed $(<4 \%)$, an entry was made to indicate that the data were missing. Only bands originating from the sensitive parent, which were absent in the resistant parent and present in both $F_{1}$ hybrids (that is heterozygous in the $F_{1}$ hybrids), were used in the linkage analysis. This resulted in a robust linkage map that allowed unambiguous integration of markers subsequently determined to be linked to loci encoding the resistance phenotype, which was recessive at the doses of phosphine used for selection. The critical value for linkage detection was $P=0.05$ ( $>95 \%$ probability that a predicted linkage was not a false positive). Recombination frequencies were converted to map distances using the Kosambi function. Any marker that caused a map expansion of $>3.0 \mathrm{cM}$ was tested for scoring errors. Such markers were found, but upon rechecking the scoring was deemed to be accurate.

\section{Cloning and sequencing of rp5.11: a marker for the high-level resistance gene from QRD569}

A DNA fingerprint was created for the sensitive female parent using primer RP5 (5'-TGCTGGTTAC-3'). The dried polyacrylamide gel was aligned with the autoradiography film and the DNA band corresponding to marker rp5.11 of the sensitive female parent was excised and ligated into $t$-tailed pBluescript vector, transformed into DH5 $\alpha$ Escherichia coli chemically competent cells and plated on LB medium containing $25 \mu \mathrm{g} \mathrm{ml}^{-1}$ ampicillin. Colonies were screened for inserts and promising clones were sequenced. Specific primers were then designed with which to amplify the cloned fragments.
Marker confirmation and conversion to a co-dominant STS marker

The marker sequence was confirmed by specific amplification using a modified PCR protocol. Each reaction volume of $20 \mu \mathrm{l}$ contained: $2 \mu \mathrm{l} 10 \times$ PCR buffer $(100 \mathrm{mM}$ Tris- $\mathrm{HCl}, \mathrm{pH} 8.0,100 \mathrm{mM} \mathrm{KCl}, 50 \mathrm{mM} \mathrm{MgCl} 2), 2 \mu \mathrm{l} 1 \mathrm{mM}$ dNTPs, $0.5 \mu \mathrm{l} 10 \mathrm{U}_{\mu} \mathrm{l}^{-1}$ Taq DNA polymerase (Perkin Elmer), $1 \mu \mathrm{l}$ each of a $10 \mu \mathrm{M}$ stock of forward and reverse primers (specific to each clone) as well as $1 \mu \mathrm{l}$ of 1:10 diluted template DNA stock. The PCR reaction was preincubated at $94{ }^{\circ} \mathrm{C}$ for $2 \mathrm{~min}$; then subjected to 35 cycles of $94{ }^{\circ} \mathrm{C}$ for $30 \mathrm{~s}, 55^{\circ} \mathrm{C}$ for $30 \mathrm{~s}, 72{ }^{\circ} \mathrm{C}$ for $1 \mathrm{~min}$; with a final extension of $72^{\circ} \mathrm{C}$ for $2 \mathrm{~min}$. The polymorphic DNA fragment corresponding to rp5.11 was validated by comparison against the mapping population to allow the amplified DNA to be placed on the published genetic linkage map for $R$. dominica (Schlipalius et al., 2002). Following confirmation that the primers amplified a DNA marker specific to the rp5.11 locus, the marker was deemed to be a sequence-tagged site (STS), and was renamed STS5.11.

\section{Fine-scale mapping of rph2}

Fine-scale mapping was carried out to allow the distance between marker STS5.11 and the resistance gene to be estimated. This was achieved by testing $170 \mathrm{~F}_{10}$ beetles exposed to $1.0 \mathrm{mgl}^{-1}$ phosphine and a total of 419 $\mathrm{F}_{20}$ beetles surviving exposure to $0.5 \mathrm{mg} \mathrm{l}^{-1}$ phosphine for $48 \mathrm{~h}$. Both of these doses resulted in survival of individuals only if they were homozygous for the rph2 resistance allele near the STS5.11 locus (Schlipalius et al., 2002). Surviving individuals not homozygous for the resistance allele of STS5.11 were scored as recombinant and the approximate map distance between $r p h 2$ and the STS5.11 marker was estimated using the formula: $\mathrm{cM}=($ number of recombination events $\times 100) /($ number of chromosomes analysed $\times$ (number of generations/2)) . This formula derives approximate map distances from the accumulated recombination events over multiple generations.

\section{STS5.11 determination of $F_{5}$ progeny of the QRD14 $\times$ QRD569 intercross}

Since the marker STS5.11 is co-dominant, it allowed detailed analysis of the genotypes of $\mathrm{F}_{5}$ beetles selected at a wide range of phosphine doses. The $\mathrm{F}_{5}$ progeny of the sensitive (QRD14) female $\times$ resistant (QRD569) male intercross (described above) was fumigated at 0.001, $0.003,0.004,0.005,0.01,0.05,0.1,0.3,0.4,0.5$ and $1.0 \mathrm{mg}^{-1}$ phosphine, and the STS5.11 genotype of the survivors was determined. Combination of this data set with previous genotype determination at the rph1 locus (Schlipalius et al., 2002) allowed the resistance phenotype to be measured for a wide range of allelic combinations.

\section{Fitness cost analysis of $r p h 2^{r}$}

The marker STS5.11 was also used to investigate the fitness costs associated with $r p h 2^{r}$ (resistant) and $r p h 2^{s}$ (sensitive) alleles. For this analysis, the genotypes of 99, 92 and 96 individuals from an unselected population of the $F_{5}, F_{10}$ and $F_{20}$ progeny of a cross between a QRD14 female and a QRD569 male were determined. This population was a sibling line to that previously used to 
establish the $R$. dominica genetic map (Schlipalius et al., 2002).

\section{Results}

\section{Intercross linkage mapping of the moderately resistant strain QRD369}

The linkage map of the intercross between moderately resistant (QRD369) and sensitive (QRD14) strains contains 23 linked markers in six linkage groups (LGs) with an additional 16 unlinked markers, an average distance between linked markers of $9.9 \mathrm{cM}$ and an estimated $58 \%$ of the genome contained between linked markers. The linkage map is shown in Figure 1. If one conservatively assumes that the unlinked markers provide an equivalent coverage of the genome $(\sim 10 \mathrm{cM}$ each), they should cover an extra $160 \mathrm{cM}$ (or $40 \%$ ) of the genome. Therefore, $\sim 98 \%$ of the genome would have been scanned for linked resistance loci from QRD369 using the intercross population.

\section{Identification of a resistance locus on LG6 in strain QRD369}

Polymorphic DNA fragments in the QRD14 $\times$ QRD369 intercross that co-migrated unambiguously with specific

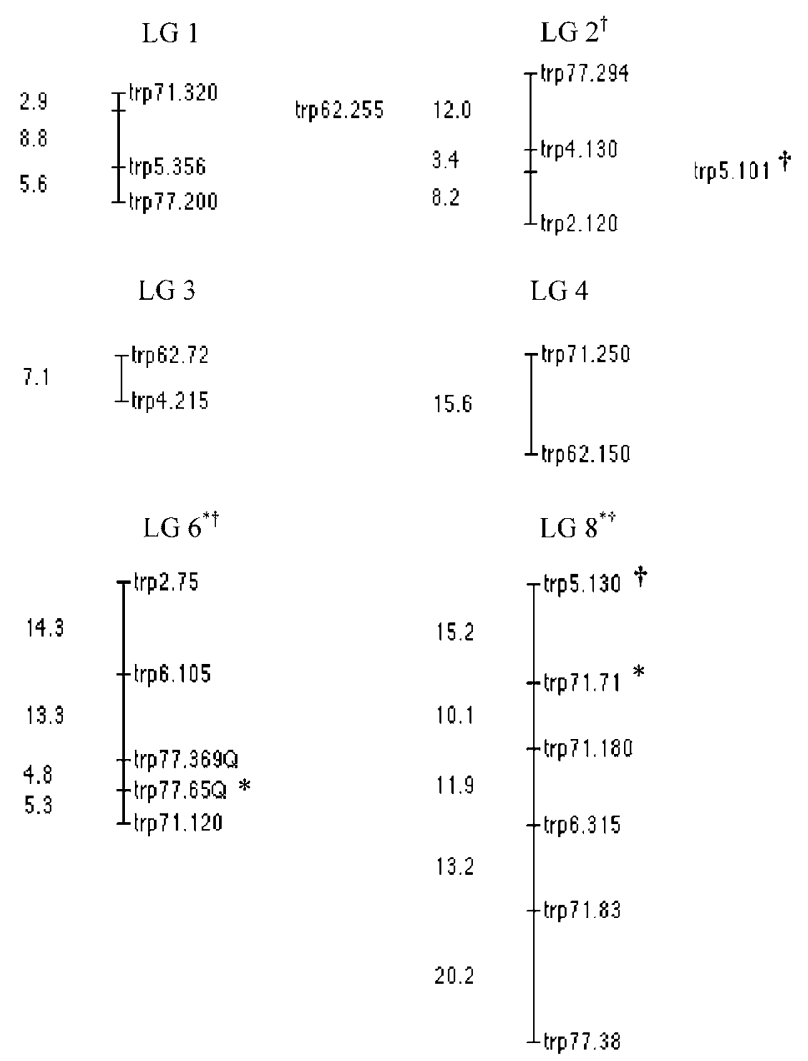

Figure 1 The genetic linkage map of Rhyzopertha dominica intercross between a moderate resistance strain (QRD369) and a susceptible strain (QRD14) consisting of 23 random amplified DNA fingerprinting (RAF) markers in six linkage groups (LGs) with a total length of $172.1 \mathrm{cM}$. An asterisk $\left(^{*}\right)$ denotes a marker with common identity to the previously published map of the QRD14 $\times$ QRD569 intercross. A dagger $(\dagger)$ denotes common identity with the backcross map (Figure 2). Markers tightly linked to resistance locus are labelled with ' $\mathrm{Q}$ '. bands in the strong resistance intercross, QRD14 $\times$ QRD569, allowed some LGs to be named consistently between the genetic map and that published in Schlipalius et al. (2002) and are marked with an asterisk $\left({ }^{*}\right)$ in Figure 1. Names of markers on the current map consist of the acronym trp (intercross resistance primer) followed by the primer number, a dot and the distance of migration of the DNA fragment from the origin on the polyacrylamide gel in millimetres.

LG6 of the QRD369 intercross map was characterized by two markers (trp77.65 and trp77.369), which co-migrated with two markers from LG6 of the previously published QRD569 map (rp77.49 and rp77.337) (Schlipalius et al., 2002). There are five markers in LG6 of the QRD369 map in the following order: trp2.75, trp6.105, $\operatorname{trp} 77.369, \operatorname{trp} 77.65$ and trp71.120. A second group of $80 \mathrm{~F}_{4}$ individuals that survived selection at $0.04 \mathrm{mg} \mathrm{l}^{-1}$ phosphine was then used for high-resolution mapping with respect to the resistance locus. Markers trp6.105, trp77.369, trp77.65 and trp71.120 were determined to be $3.7,<0.1,0.9$ and $2.5 \mathrm{cM}$ from the resistance locus, respectively (Figure 1). In fact, no recombination events were observed between trp77.369 and the resistance gene, so the figure of $0.1 \mathrm{cM}$ is an upper estimate of the genetic distance. No resistance loci, other than that identified by marker trp77.369 were found in QRD369, despite the fact that the 39 polymorphic markers provided on average one marker every $10.0 \mathrm{cM}$ across $98 \%$ of the genome.

\section{No major resistance locus on LG5}

We analysed a third group consisting of $42 \mathrm{~F}_{8}$ beetles from the same QRD14 $\times$ QRD369 intercross to confirm the existence of the rph1 resistance in QRD369 and to test the strength of the association at a range of doses. A combined total of 25 survivors at the lower doses of phosphine, five each from many survivors at each dose $\left(0.007,0.01,0.015,0.02\right.$ and $\left.0.05 \mathrm{mg} \mathrm{l}^{-1}\right)$, three survivors at $0.07 \mathrm{mg}^{-1}$ and $14 \mathrm{~F}_{8}$ beetles that died at $0.007 \mathrm{mgl}^{-1}$ were subjected to DNA fingerprinting using primers RP6 (5'-TGCTGGTTTCC-3') and RP77 (5'-GAGGTTTCAG-3'). It was observed that no survivor contained a band corresponding to the sensitive allele of marker trp77.369 and that the allele was present in all dead individuals. Survivors from all doses therefore must have been homozygous for the resistance allele present on LG6 and there was no evidence for a second resistance locus contributing to the phenotype at any of the doses. That is, the resistance allele on LG6 was significantly associated with resistance at each dose $\left(\chi^{2}=5, P=0.025\right.$, d.f. $\left.=1\right)$ and strongly associated with resistance at all doses $\left(\chi^{2}=14, P=0.0002\right.$, d.f. $\left.=1\right)$, and there was no weaker association at any of the lower doses, that would indicate evidence for a major secondary resistance factor similar to that seen in strain QRD569. If there are any secondary factors that contribute to the resistance in QRD369, their effects are very minor and below the limit of detection in this study

\section{Backcross linkage mapping of the moderately resistant strain QRD369}

A backcross population was produced by backcrossing an $F_{1}$ sibling of the female used for the intercross to the resistant male parent from the QRD369 strain. The map 

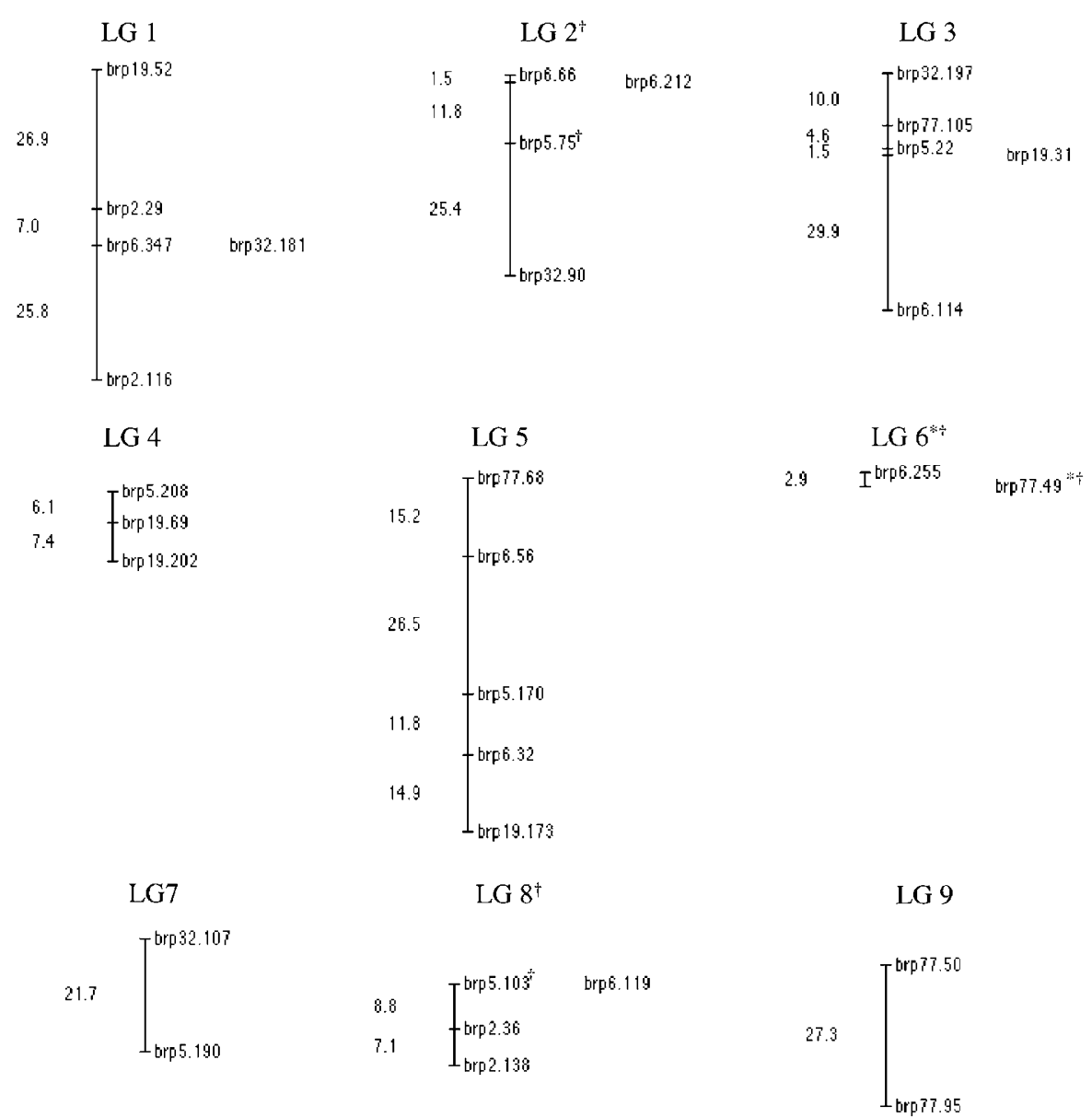

Figure 2 The genetic linkage map of $R$. dominica backcross between a moderate resistance strain (ORD369) and a susceptible strain (QRD14) consisting of 32 random amplified DNA fingerprinting (RAF) markers in nine linkage groups (LGs) with a total length of $294 \mathrm{cM}$. An asterisk $(*)$ denotes a marker with common identity to the previously published map of the QRD14 $\times$ QRD569 intercross. A dagger $(\dagger)$ denotes common identity with the intercross map (Figure 1).

from this backcross population contains 32 markers in nine LGs plus an additional four unlinked markers, corresponding to an average distance between linked markers of $10.8 \mathrm{cM}$ (Figure 2). The estimated map size is $345 \mathrm{cM}$, which represents $89 \%$ coverage of the genome (Schlipalius et al., 2002), an estimate that approaches $100 \%$ if the unlinked markers are included in the calculation. Because individuals in the mapping population were assessed for resistance at either 0.015 or $0.04 \mathrm{mg} \mathrm{l}^{-1}$ phosphine, the location of the resistance gene could be assessed directly from the mapping population. A total of 24 of 46 individuals survived exposure to the low dose and 22 of 45 individuals survived exposure to the high dose. The observed $\sim 50 \%$ survival rate at both doses is consistent with a previous publication that identified a single major resistance gene in strain QRD369 (Collins et al., 2002). Thus, one could assume the survivors were homozygous for the major resistance gene, whereas the dead beetles could be assumed to be heterozygous. Two markers were found that were linked to a resistance locus, namely brp6.255 and brp77.49 (the naming convention uses brp as an acronym for backcross resistance primer, followed by the primer number and the distance migrated on the gel). Because the intercross and backcross populations were derived from the same initial cross between QRD14 and QRD369, common markers could be identified with a great deal of certainty. For example, the marker brp77.49 is the same maternally derived marker as trp77.63 that was previously found to be linked to a resistance factor on LG6 in the intercross. The marker brp77.49 also co-migrates with rp77.49, which was linked to rph1 in the highly resistant strain (Schlipalius et al., 2002). In the backcross population, the genetic distance between marker brp77.49 and the resistance locus is $\sim 1.1 \mathrm{cM}$ (Figure 2). This is due to a single detected recombination event and is the maximum resolution possible in the backcross.

It is of interest that a paternal marker (band from the resistant male parent) brp6.79 was also putatively linked to resistance. This marker co-migrated precisely with rp6.79, a marker identified as closely linked to the resistance locus rph1 on LG6 in strain QRD569 as determined in the original intercross (Schlipalius et al., 2002).

We determined that the marker was linked to resistance because if the inherited DNA band were a neutral marker, that is unlinked to resistance, we would have expected a 1:1 segregation ratio in both sensitive and resistant individuals. However in a group of 46 resistant backcross offspring, the observed ratio was 1:0 and this allowed us to strongly reject the hypothesis that this marker is unlinked to a resistance locus $\left(\chi^{2}=22\right.$, $\left.P<2.73 \times 10^{-6}\right)$. 


\begin{tabular}{|c|c|c|}
\hline $\mathrm{P}_{0}$ & Male $-_{r}+_{r}$ & Female $-\mathrm{s}^{+} \mathrm{s}$ \\
\hline BC & $\mathrm{P}_{0}$ Male (as above) & $\mathrm{F}_{1}$ female $-\mathrm{s}^{+}{ }_{\mathrm{r}}$ \\
\hline $\mathrm{BC}$ progeny & $\begin{array}{l}\text { sensitive }-{ }^{-}{ }_{r} \text { OR - } \\
s^{-}\end{array}$ & resistant $+_{r}+{ }_{r} \mathbf{O R}-{ }_{r}+_{r}$ \\
\hline
\end{tabular}

Figure 3 Proposed model of inheritance of the paternal marker brp6.79 in the QRD14 $\times$ QRD369 backcross. An important marker was found in the backcross population that unlike other markers in the study was dominant and paternally inherited rather than recessive and maternally inherited. Furthermore, the backcross parent appeared to be heterozygous for the marker. In this figure, a ' + ' denotes presence of the dominant marker and allele, whereas ' - ' denotes the recessive allele (absence of marker). In the backcross progeny, a 3:1 (dominant ' + ': recessive ' - ') segregation ratio would be expected, 1:1 in sensitive individuals and 1:0 in resistant individuals, which is indeed the case as discussed in the text.

The alternative explanation that fits the data is that all 46 resistant backcross offspring inherited the dominant paternal brp6.79 marker via the $\mathrm{F}_{1}$ female. Fortuitously, the backcross male was heterozygous for the dominant marker $\left(-_{\mathrm{r}}+_{\mathrm{r}}\right)$. The $\mathrm{F}_{1}$ female was also heterozygous for the marker $(-\mathrm{s}+\mathrm{r})$, so had inherited the allele for absence of the marker $(-s)$ from the sensitive maternal parent. The sensitive offspring likewise inherited the recessive marker $(-\mathrm{S})$ from the $\mathrm{F}_{1}$ female and either $\left(-_{r}\right)$ or $\left(+_{r}\right)$ from the backcross male (Figure 3 ).

As a result, it was possible to unambiguously determine the brp6.79 genotype of one quarter of the progeny, those that were $\left(-_{-}-\mathrm{r}\right)$, having inherited the maternal $\left({ }_{S}\right)$ marker (lack of a band) from the $F_{1}$ female together with the $\left(-_{r}\right)$ marker (lack of a band) from the backcross male. Such individuals would be expected to be heterozygous for the linked recessive resistance gene and thus sensitive to phosphine, if the marker was actually linked to the resistance gene as predicted. Indeed, all individuals that lacked the DNA fragment $\left(-{ }_{S}-\mathrm{r}\right)$ were sensitive to phosphine. Combining the sensitive and resistant groups together, the segregation ratio was the expected 3:1 under the scenario outlined in Figure 3, with the marker segregating at $\sim 1: 1$ in the sensitive individuals. Considering that $100 \%$ of the resistant progeny had this DNA band in their fingerprints, and we thus determined the marker to be linked to a phosphine-resistant gene, most likely rph1.

\section{Cloning and confirmation of marker rp5.11}

We have focussed our efforts on the analysis of the rph2 resistance locus in line QRD569 for the following reasons: First, the intercross and backcross (both paternal and maternal) markers indicated that the moderate resistance of QRD369 was due to the rph1 gene on LG6. As there was no evidence to support the notion of a resistance factor corresponding to rph2 on LG5, or indeed anywhere else in the genome, rph1 must be solely responsible for the vast majority of moderate level resistance. The genetics of moderate level resistance in strain QRD369, essentially a study of the rph1 gene, already has been well described (Collins et al., 2002). Second, rph2 is responsible for high-level resistance towards phosphine and is therefore of considerable economic significance.

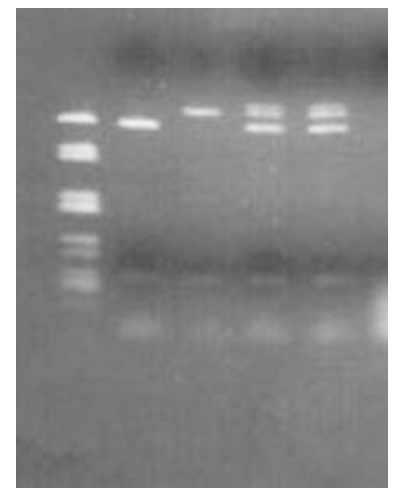

Figure 4 Agarose electrophoresis gel of marker STS5.11 showing clearly distinguishable genotypes. Lane 1, DNA size marker pBluescript/EcoRV, lane 2, $\mathrm{P}_{0}$-resistant male (homozygous resistant); lane 3, $\mathrm{P}_{0}$-susceptible female (homozygous susceptible); lanes 4 and $5, \mathrm{~F}_{1}$ hybrid progeny.

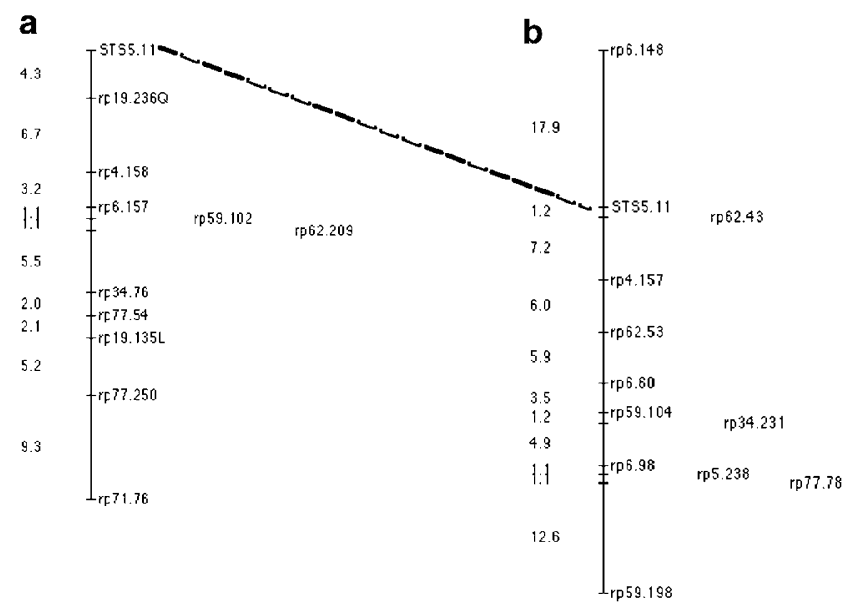

Figure 5 Linkage group five (LG5) of $R$. dominica using (a) maternally inherited markers and (b) paternally inherited markers, showing the co-dominant inheritance of STS5.11 in the strong resistance intercross $(\mathrm{QRD} 14 \times \mathrm{QRD} 569)$.

The STS marker STS5.11 is a STS converted from the dominant marker rp5.11 at the rph2 locus on LG5. STS5.11 was confirmed by specific amplification as shown in Figure 4. Subsequent linkage analysis revealed that STS5.11 did indeed map to the same locus as rp5.11 using both maternally and paternally derived markers in the QRD14 $\times$ QRD569 intercross mapping population (Figure 5). In the LG created from paternally derived markers we see markers flanking the resistance locus STS5.11, which suggests that this locus is not in a region of suppressed recombination on the end of a chromosomal arm. This result compensates for the fact that we have not found any maternally derived markers that flank this locus and suggests that the linkage map already reported for $R$. dominica (Schlipalius et al., 2002) is not quite saturated.

The nucleotide sequence of STS5.11 is shown in Figure 6. The marker is $673 \mathrm{bp}$ in length, with the resistant polymorphism being an $80 \mathrm{bp}$ deletion between position 333 and 412 inclusive on the marker sequence. A blastx search of the GenBank database using the STS5.11 sequence as a query showed that part of the marker 


\section{R. dominica PQISNDPKNQYIRYELDYVL* \\ P+I ND KN YIRYELDY+L \\ Homo sapiens PRIYNDDKNTYIRYELDYIL*}

Figure 6 Sequence of marker STS5.11. The nucleotide sequence was used to query the non-redundant amino-acid sequence database of GenBank using the blastx algorithm. A candidate exon was identified in STS5.11 that was homologous to a fragment of the spliceosomal subunit $3 \mathrm{~b}$ from Homo sapiens. Underlined nucleotides in bold type correspond to the primer sequences. Nucleotides in italics indicate the deleted portion of the marker in the resistance genotype. The asterisk ${ }^{*}$ ) denotes a translation stop codon.

sequence had a high homology to a fragment of premRNA splicing factor subunit SF3b from Homo sapiens (Figure 6).

\section{Fine-scale mapping of $r p h 2$}

The effective use of STS5.11 as a marker for resistance is dependent on it being very tightly linked to the rph2 resistance gene. The ability to score STS5.11 as a codominant size polymorphism allowed us to rapidly determine the degree of linkage between it and rph2. Only one recombination event has been observed between the marker and rph2 out of 170 phosphineresistant $\mathrm{F}_{10}$ beetles, and no recombination events were observed out of 419 resistant $\mathrm{F}_{20}$ beetles. We combined these data sets to estimate the map distance between STS5.11 and rph2, by normalizing the number of recombination events observed in $\mathrm{F}_{20}$ and $\mathrm{F}_{10}$ beetles according to the number of generations that had elapsed.

Thus, using the formula: $\mathrm{cM}=(\mathrm{R} \times 100) /\left(\mathrm{C}_{\mathrm{F} 10} \mathrm{G}_{\mathrm{F} 10} /\right.$ $\left.2+\mathrm{C}_{\mathrm{F} 20} \mathrm{G}_{\mathrm{F} 20} / 2\right)$; where $\mathrm{R}=$ the number of recombinations, $\mathrm{C}=$ the number of chromosomes analysed and $\mathrm{G}=$ the number of generations; we estimated that the approximate distance between STS5.11 and rph2 was $<0.01 \mathrm{cM}$, or $\sim 12 \mathrm{kbp}$ using previously estimated values of physical distance to recombination frequency (Schlipalius et al., 2002).

\section{Genotype-phenotype comparison of the highly resistant strain QRD569}

Having directly confirmed rph1 as the genetic background in which the resistance allele of rph2 was selected, we then wished to identify possible interactions between the two genes. The co-dominance of marker
STS5.11 and its extremely tight linkage to $r p h 2$ made it possible to unambiguously determine the resistance genotypes at this locus in $\mathrm{F}_{5}$ beetles selected at various doses of phosphine. This allowed us to identify resistance thresholds of individuals homozygous or heterozygous for rph2 (Table 1) and observe semidominant effects of the resistance allele as well. In the F5 population four individuals survived at $0.01 \mathrm{mg} \mathrm{l}^{-1}$ or above, despite having a genotype exhibiting susceptibility at rp6.79. Of these, two exhibit recombination between the markers that flank rp6.79. It is reasonable to hypothesize that these recombination events separate the marker rp6.79 from the resistance gene. The remaining two survivors exhibiting a susceptible genotype may have been due to two recombination events within the 5.5-cM interval over four generations giving the appearance of no recombination. Thus, they have been counted as 'resistant' at the rp6.79 locus.

In this analysis it is useful to refer to a resistance threshold, which is defined as the minimum dose of phosphine at which a particular resistance allele must be present (either homozygous or heterozygous) for an individual to survive. The threshold concentration for the resistance allele linked to rp6.79 $\left(r p h 1^{r r}\right)$ is between 0.05 and $0.1 \mathrm{mg}^{-1}$ for a 48 -h exposure at $25^{\circ} \mathrm{C}$. The threshold concentration for the resistance allele linked to STS5.11 in either its heterozygous or homozygous state is between 0.01 and $0.05 \mathrm{mgl}^{-1}$, although it is clear that homozygosity confers a greater resistance. However when combined with the resistance allele at rp6.79 in its homozygous state $\left(r p h 1^{r r}\right)$, the threshold for heterozygous STS5.11 $\left(r p h 1^{r r}+r p h 2^{r s}\right)$ is between 0.1 and $0.2 \mathrm{mg} \mathrm{l}^{-1}$, whereas the threshold is $>1.0 \mathrm{mg} \mathrm{l}^{-1}$ (Table 1) when both genes are homozygous for resistance. Given 
Table 1 Summary of genotype analysis of $F_{5}$ survivors of phosphine fumigation

\begin{tabular}{|c|c|c|c|c|c|c|c|c|c|c|c|c|}
\hline \multicolumn{2}{|c|}{ genotype } & \multicolumn{11}{|c|}{ dose of phosphine $(\mathrm{mg} / \mathrm{l})$} \\
\hline rp6.79 & rp5.11 & unselected & 0.003 & 0.004 & 0.005 & 0.01 & 0.05 & 0.1 & 0.2 & 0.3 & 0.4 & 1.0 \\
\hline $\begin{array}{l}\mathrm{S}^{++} \\
\text {or } \\
\mathrm{S}^{+}\end{array}$ & ss & 32 & 18 & 0 & 1 & 0 & 2 & 0 & 0 & 0 & 0 & 0 \\
\hline $\begin{array}{l}S^{+*} \\
\text { or } \\
S^{+}\end{array}$ & rs & 27 & 26 & 14 & 4 & 1 & 0 & 2 & 0 & 0 & 0 & 0 \\
\hline $\begin{array}{l}\mathrm{S}^{++} \\
\text {or } \\
\mathrm{S}^{+}\end{array}$ & $r r$ & 10 & 10 & 3 & 7 & 9 & 0 & 0 & 0 & 0 & 0 & 0 \\
\hline $\mathrm{R}^{+}$ & ss & 6 & 6 & 3 & 2 & 8 & 5 & 0 & 0 & 0 & 0 & 0 \\
\hline $\mathrm{R}^{+}$ & rs & 21 & 21 & 7 & 6 & 5 & 16 & 10 & 0 & 0 & 0 & 0 \\
\hline $\mathrm{R}^{+}$ & $r r$ & 4 & 4 & 5 & 2 & 0 & 5 & 7 & 10 & 10 & 8 & 44 \\
\hline $\begin{array}{l}\text { No of } \\
\text { ans }\end{array}$ & & 100 & 84 & 32 & 22 & 23 & 32 & 19 & 10 & 10 & 8 & 44 \\
\hline Total & ested & 100 & 100 & 100 & 100 & 100 & 300 & 400 & 750 & 500 & 500 & 7850 \\
\hline
\end{tabular}

Summary of genotype analysis of $\mathrm{F}_{5}$ survivors of phosphine fumigation showing relative ratio of the genotypes in the surviving beetles at the various doses. ' $\mathrm{R}$ ' indicates homozygous for the resistant allele and ' $\mathrm{S}$ ' indicates heterozygous or homozygous for the susceptible allele. For marker STS5.11, ' $r r^{\prime}$ and 'ss' denote homozygous for the resistant and susceptible allele, respectively.

${ }^{a}$ Data obtained from $F_{2}$ mass cross (bulk segregant) analysis. The heavy line indicates threshold values of phosphine exposure separating phenotypes. Figures are expressed as the actual number of beetles analysed for each dose. Figures in bold type indicate genotypes of four individuals discussed in further detail in the text.

Table 2 Genotypic distribution data of STS5.11 over 20 generations of progeny from a single pair cross between sensitive (QRD14) and resistant (QRD569) lines of Rhyzopertha dominica maintained in the absence of phosphine selection

\begin{tabular}{lccccc}
\hline & Homozygous resistant & Heterozygous & Homozygous sensitive & Total progeny analysed & Significance $^{\text {a }}$ (P-value) \\
\hline F5 & 14 & 48 & 37 & 99 & 0.80 \\
F10 & 20 & 36 & 36 & 92 & 0.064 \\
F20 & 16 & 46 & 34 & 96 & 0.94 \\
\hline
\end{tabular}

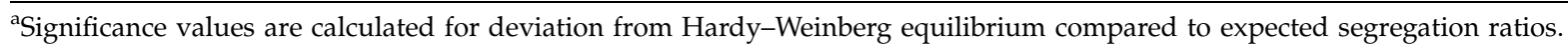

that the $\mathrm{LD}_{99.9}$ for the fully susceptible phenotype is $0.004 \mathrm{mg} \mathrm{l}^{-1}$, we estimated that the resistance alleles of rph1 and rph2 have individual contributions towards resistance that lie in the ranges of $12.5 \times<r p h 1^{r r}<25 \times$ $2.5 \times<r p h 2^{r s / r r}<12.5 \times$. While the semi-dominant effect of $r p h 2^{r s}$ is clearly seen when combined with $r p h 1^{r r}$, giving a range of $25 \times<r p h 1^{r r}, r p h 2^{r s}<50 \times$. For both resistance alleles together the resistance factor is $>250 \times$. Once again, it is clear that the strong synergistic interaction occurs only when rph1 and rph2 are both homozygous for resistance and that $r p h 2$ has semidominant effects.

\section{Relative fitness of rph2}

The co-dominance of marker STS5.11 allowed us to analyse deviation from Hardy-Weinberg equilibrium in the distribution of genotypes as well as persistence of the rph 2 resistance allele over 15 generations (from $F_{5}$ to $F_{20}$ ) in the absence of selective pressure (Table 2). The $\chi^{2}$-values for the $\mathrm{F}_{5}\left(\chi^{2}=0.06, P>0.80\right.$, d.f. $\left.=1\right)$ and $\mathrm{F}_{20}$ $\left(\chi^{2}=0.004, P>0.94\right.$, d.f. $\left.=1\right)$ generations indicated no deviation from Hardy-Weinberg equilibrium throughout these generations. The $F_{10}$ generation showed an increase in the homozygous-resistant genotype $\left(\chi^{2}=3.43, P>0.064\right.$, d.f. $\left.=1\right)$, nearly reaching the statistical significance threshold of $P \leqslant 0.05$, but this deviation did not persist, indicating that it represents random flux in allele frequencies in the population. With regard to the persistence of the resistance allele, no significant differences in the allele frequencies were observed between the $F_{5}$ and $F_{10},\left(\chi^{2}=0.663, P=0.415\right.$, d.f. $\left.=1\right), F_{5}$ and $\mathrm{F}_{20},\left(\chi^{2}=0.408, P=0.523\right.$, d.f. $\left.=1\right)$ or the $\mathrm{F}_{10}$ and $\mathrm{F}_{20}$, $\left(\chi^{2}=0.0365, P=0.848\right.$, d.f. $\left.=1\right)$ generations.

\section{Discussion}

\section{Evolution of resistance}

We previously determined that a strain of $R$. dominica QRD569 that is resistant to high levels of the fumigant phosphine contains two resistance genes, one on LG5, rph2, and the other on LG6, rph1, which together act synergistically to confer resistance $>250 \times$ the basal level of tolerance in a fully sensitive strain. Synergistic (greater than additive) gene interactions have been noted previously in pesticide-resistant strains of insects (Arnold and Whitten, 1976; Raymond et al., 1989; Shono et al., 2002). However, examples of field-collected strains containing synergistically interacting genes are very rare. 
The synergistic interaction of resistance genes clearly seen in $R$. dominica, combined with the unique mode of action of phosphine, provides an opportunity to understand how such genetic interactions contribute to the evolution of pesticide resistance.

We have now examined a moderately resistant predecessor of the highly resistant strain isolated from the same geographical region of Australia that exhibits resistance $>31 \times$ that of the fully sensitive strain (Collins et al., 2002). We have determined that moderate resistance was achieved by means of a single major resistance allele, which is consistent with the observations of Collins et al. (2002). Furthermore, we have determined that high-level resistance was not achieved by a secondary mutation in the moderate resistance gene, rph1, which created a stronger resistance allele at the same locus, but rather by acquisition of a resistance allele at a second locus, rph2, that was not found to be a significant contributor to resistance in the moderately resistant line. Thus, high-level resistance arose via stepwise acquisition of a resistance allele at each of two distinct loci. These two resistance genes actually account for most, but not all of the high-level resistance phenotype, with additional genes of minor effect being implicated in the residual resistance. It is possible that these minor loci are not fixed in the resistant parental strains used in this study and thus may have been lost due to a 'founder effect' by pairwise crossing of individual insects, or they may be polygenic in nature, that is many genes of very minor effect that are selected for during the rounds of selection required for fixation of the major genes. In either case, loci corresponding to such minor genes have not been identified by this analysis.

\section{Fine-scale mapping}

Because the phenotype of the moderately resistant strain of $R$. dominica had already been characterized in detail (Collins et al., 2002), we proceeded to analyse the second locus, rph2, which was responsible for the high-level resistance. Cloning of the marker DNA fragment at STS5.11 provided a high throughput co-dominant marker which, combined with the reproductive capacity of the beetle, allowed extremely high-resolution mapping of rph2. The co-dominant STS5.11 marker is so tightly linked to $r p h 2(\leqslant 0.01 \mathrm{cM})$ that we can now unambiguously determine the resistance genotype of an individual at this locus.

\section{Detailed genotype/phenotype of QRD569}

Genetic dominance or recessiveness influences the rate of selection (or fixation) and is one of the most important determinants of the rate of development of resistance in the field (Gazzoni, 1998). The co-dominant marker STS5.11 facilitated detailed phenotypic analysis of the resistance allele of $r p h 2$, in particular the semi-dominance, as well as the genetic interactions between $r p h 2$ and rph1. For example, coupled genotypic and phenotypic analysis was carried out on the segregating progeny of an intercross between highly resistant and fully sensitive strains. The resistance allele of $r p h 2$ showed an incompletely recessive effect when heterozygous as well as a synergistic effect with $r p h 1$ when both were homozygous for their respective resistance alleles. This synergism of the rph2 resistance allele would have allowed fully resistant individuals to easily survive fumigation, significantly contributing to the evolution of resistance in the field.

Resistance management strategies such as refuges are based on the continual reintroduction of dominantsensitive alleles into a population. In this strategy, rare homozygous resistant survivors of pesticide treatment are likely to mate with homozygous-sensitive individuals from the refuges, resulting in the generation of sensitive heterozygous progeny (Georghiou and Taylor, 1977). In contrast, dominant resistance alleles cannot be managed by such a strategy, as the resistance phenotype will not be masked. A semi-dominant resistance phenotype can be selected rapidly, as heterozygotes have a selective advantage over sensitive individuals, while fully resistant homozygotes have a further advantage over heterozygotes. In the case of semi-dominance, however, management of resistance as a recessive trait is still possible. The management tactic of using mixtures of pesticides relies on at least a functional recessiveness in that only resistant homozygotes should survive exposure to the pesticides used (Tabashnik, 1990; Hoy, 1998), thus maintaining the rarity of the resistance alleles.

\section{Mechanism of selection for resistance in the field}

Resistance genetics modelling suggests that strong selection pressure will favour single genes with a strong resistance effect (a monogenic resistance response) (McKenzie, 1996), whereas multiple resistance genes of weak effect (a polygenic resistance response) will result if the level of selection is within the mortality distribution of the original sensitive population. Indeed, low-level phosphine resistance, from 3- to 12-fold, is quite easily selected in the laboratory (Monro et al., 1972; Saxena and Bhatia, 1980). The resistance gene from the first major resistance allele, rph1, provided a level of resistance much greater than the total polygenic effect, exactly what would be expected of a classical monogenic resistance response. In contrast, our analysis revealed that in the absence of the resistance allele at rph1, the resistance provided by rph2 was similar in magnitude to a polygenic effect. It is quite probable that fumigation to control field (polygenic) resistance during the early years of phosphine use in Australia effectively controlled the $r p h 2$ resistance allele but allowed the $r p h 1$ resistance allele to become established. The increased phosphine application rates implemented with the advent of weak resistance would have been even more effective at controlling a resistance allele at the rph2 locus.

In the Darling Downs region of Queensland, the synergistic effect of the resistance allele at $r p h 2$ produced an extremely resistant strain of $R$. dominica when combined with the rph1 allele. Furthermore, the semi-dominant phenotype of the resistance allele at rph2 would have contributed to survival of heterozygous individuals when the allele first arose. Interbreeding of surviving heterozygous beetles would have given rise to the homozygous, highly resistant progeny that were discovered in Queensland in 1997. The semi-dominance of the allele probably contributes to re-selection of the high-level resistance phenotype in grain stores that are subjected to repeated fumigation. 
Weak resistance seems to have arisen at multiple times and locations and is now ubiquitous across eastern Australia (Schlipalius et al., 2006). It is interesting to note that high-level resistance has not seemed to arise due to the acquisition of multiple resistance genes from the merger of moderately resistant populations of insects, that is in a hybrid zone. Rather, weak resistance became ubiquitous over a nearly 10-year period after which distinct foci of high-level resistance arose. The implication is that mutations in the same gene are responsible for moderate level resistance in all instances or that resistance is due to epistatic mutations in different genes, perhaps within the same biochemical pathway. Highlevel resistance has arisen at widely separated locations including two locations in Queensland, a single location in New South Wales and one in South Australia, across a range of more than $1000 \mathrm{~km}$. These growing regions are separated by desert and native habitats, and movement of grain between these regions is very rare (in Australia, most grain tends to go straight from the farm to port for export) (PJ Collins, unpublished). Thus, it is considered highly unlikely, although not impossible, that resistance arose once after which resistant insects dispersed from the site of the initial outbreak. This is important in modelling the spread of resistance and gives us an indication of the rate of evolution of resistance in independent populations (Andreev et al., 1999).

Complementation analysis has confirmed that the genes responsible for high-level resistance are the same in all the outbreaks studied (Mau, 2007), which gives us confidence that although the number of strains used in this study are small, they are representative of high-level resistance in $R$. dominica in Australia as a whole.

The rph2 locus that we have identified acts synergistically with the rph1 locus, resulting in high-level resistance. The four high-level resistance outbreaks in Australia occurred very suddenly and gave rise to dramatically elevated levels of resistance of approximately equivalent magnitude, consistent with a synergistic interaction between resistance factors. Recent analysis of these additional highly resistant strains suggests that resistance alleles at $r p h 1$ and $r p h 2$ contribute to the resistance phenotype in each case (Mau, 2007). According to models of insecticide resistance mechanism interaction, the synergistic interaction between the two phosphine-resistant genes suggests that they contribute to resistance through unique and complementary mechanisms, such as target-site resistance combined with a detoxification mechanism (Raymond et al., 1989).

\section{Fitness costs associated with high-level resistance}

Fitness costs associated with a resistance allele can affect both the rate of resistance development and the persistence of resistance in the field (McKenzie, 1996). The QRD369 strain, and thus the rph1 gene, which on its own confers moderate level resistance, has previously been shown not to have any fitness costs associated with it (Collins et al., 2001), consistent with anecdotal observations that moderate level resistance is persistent in the field. In fact, in the same study, fitness costs were not found for several phosphine-resistant species, including $S$. oryzae and $T$. castaneum. The ability to unambiguously determine the genotype at the rph2 locus has allowed us to assess Hardy-Weinberg genotype equilibrium and allele frequency. We observed no significant change in the frequency of particular allelic combinations, indicating that the alleles were indeed in Hardy-Weinberg equilibrium at the three generations that we tested $\left(\mathrm{F}_{5}, \mathrm{~F}_{10}\right.$ or $\left.\mathrm{F}_{20}\right)$. Furthermore, we observed no significant change in frequency of the two alleles at the rph 2 locus in the absence of selective pressure over 15 generations. This is similar to the observation of Collins et al. (2001) that there is no selective disadvantage to the major gene responsible for moderate level resistance, now known as rph1. The combined conclusion is that there is probably no fitness cost associated with highlevel resistance to phosphine. This is important in setting management strategies for mitigation of resistance as many strategies rely on fitness costs associated with resistance, such as rotation of chemicals (Tabashnik, 1990; Hoy, 1998) or the stable zone strategy (Lenormand and Raymond, 1998). However, in the absence of fitness costs associated with resistance, it is expected that mitigation strategies that rely on these costs will be ineffective (Hoy, 1998) and that resistance will persist indefinitely in field populations.

The combined information of these studies into phosphine resistance take on special evolutionary significance given that phosphine acts indirectly through mitochondrial disruption leading to elevated oxidative stress (Bolter and Chefurka, 1990; Chaudhry and Price, 1992; Chaudhry, 1997). Adaptation to oxidative stress and management of mitochondrial function are extremely important evolutionary processes, which remain to be fully investigated. Understanding phosphine resistance and its mode(s) of action will provide unique insight into these adaptive mechanisms.

\section{Acknowledgements}

We thank Greg Daglish, Tina Lambkin, Linda Bond, Trevor Lambkin, Hervoika Pavic, Linda Kerr and Myrna Constantin for technical assistance; Julie Waldron, Ian Searle, Cameron Peace, Carl Ramage, Dion Harrison and Bernie Carroll for assistance with genetic mapping. This project was funded by the Grains Research and Development Corporation (GRS23 \& UQ10), Australian Research Council (00/ARC098G), Queensland Department of Primary Industries and Grainco Australia.

\section{References}

Andreev D, Kreitman M, Phillips TW, Beeman RW, ffrenchConstant RH (1999). Multiple origins of cyclodiene insecticide resistance in Tribolium castaneum (Coleoptera: Tenebrionidae). J Mol Evol 48: 615-624.

Arnold JTA, Whitten MJ (1976). Genetic basis for organophosphorus resistance in Australian sheep blowfly, LuciliaCuprina (Wiedemann) (Diptera, Calliphoridae). Bull Entomol Res 66: 561-568.

Benhalima H, Chaudhry MQ, Mills KA, Price NR (2004). Phosphine resistance in stored-product insects collected from various grain storage facilities in Morocco. J Stored Prod Res 40: 241-249.

Bolter CJ, Chefurka W (1990). The effect of phosphine treatment on superoxide dismutase, catalase and peroxidase in the granary weevil, Sitophilus granarius. Pestic Biochem Physiol 36: $52-60$ 
Chaudhry MQ (1997). A review of the mechanisms involved in the action of phosphine as an insecticide and phosphine resistance in stored-product insects. Pestic Sci 49: 213-228.

Chaudhry MQ, Price NR (1992). Comparison of the oxidant damage induced by phosphine and the uptake and tracheal exchange of phosphorus-32-radiolabelled phosphine in the susceptible and resistant strains of Rhyzopertha dominica (F.) (Coleoptera: Bostrychidae). Pestic Biochem Physiol 42: 167-179.

Collins PJ (1998). Resistance to grain protectants and fumigants in insect pests of stored products in Australia. In: Banks HJ Wright EJ, Damcevski KA (eds). Stored Grain in Australia. Proceedings of the Australian Post-Harvest Technical Conference. CSIRO: Canberra, Australia, pp 55-57.

Collins PJ, Daglish GJ, Bengston M, Lambkin TM, Pavic H (2002). Genetics of resistance to phosphine in Rhyzopertha dominica (F.) (Coleoptera: Bostrichidae). J Econ Entomol 95: 862-869.

Collins PJ, Daglish GJ, Nayak MK, Ebert PR, Schlipalius DI, Chen $W$ et al. (2001). Combating resistance to phosphine in Australia. In: Donahaye EJ, Navarro S, Leesch JG (eds). International Conference on Controlled Atmosphere and Fumigation in Stored Products. Executive Printing Services: Fresno, CA, pp 593-607.

Daglish GJ, Collins PJ (1998). Improving the relevance of assays for phosphine resistance. In: Zuxun J, Quan L, Yongsheng L, Xianchang $\mathrm{T}$ and Lianghua $\mathrm{G}$ (eds). Proceedings of the 7 th International Working Conference on Stored Product Protection. Sichuan Publishing House of Science and Technology: Beijing, China, pp 584-593.

Gazzoni DL (1998). Modeling insect resistance to insecticides using velvetbean caterpillar (Anticarsia gemmatalis) as an example. Pestic Sci 53: 109-122.

Georghiou GP, Taylor CE (1977). Operational influences in evolution of insecticide resistance. J Econ Entomol 70: 653-658.

Hoy MA (1998). Myths, models and mitigation of resistance to pesticides. Philos Trans R Soc Lond B Biol Sci 353: 1787-1795.

Lenormand T, Raymond M (1998). Resistance management: the stable zone strategy. Proc R Soc Lond B 265: 1985-1990.

Lorini I, Collins PJ, Glish G, Nayak MK, Pavic H (2007). Detection and characterisation of strong resistance to phosphine in Brazilian Rhyzopertha dominica (F.) (Coleoptera: Bostrychidae). Pest Manag Sci 63: 358-364.

Manly KF, Olson JM (1999). Overview of QTL mapping software and introduction to Map Manager QT. Mamm Genome 10: 327-334.

Mau Y (2007). Comparative genetic and toxicological analysis of phosphine resistance in the lesser grain borer, Rhyzopertha dominica (F.) (Coleoptera: Bostrichidae). PhD thesis University of Queensland: St Lucia, Australia.

McKenzie JA (1996). Ecological and Evolutionary Aspects of Insecticide Resistance. R.G. Landes Company: Austin, TX.

Mills KA (1983). Resistance to the fumigant hydrogen phosphide in some stored product species associated with repeated inadequate treatments. Mitteilungen der Deutschen Gesellschaft für allgemeine und angewandte Entomologie 4: 98-101.
Monro HAU, Upitis E, Bond EJ (1972). Resistance of a laboratory strain of Sitophilus granarius (L.) (Coleoptera, Curculionidae) to phosphine. J Stored Prod Res 8: 199-207.

Nayak MK, Collins PJ, Pavic H (2003). Developments in phosphine resistance in China and possible implications for Australia. In: Wright EJ, Webb MC and Highley E (eds). Stored Grain in Australia 2003. Proceedings of the Australian Postharvest Technical Conference. CSIRO Stored Grain Research Laboratory: Canberra, Australia, pp 156-159.

Rajendran S, Narasimhan KS (1994a). Phosphine resistance in the cigarette beetle Lasioderma serricorne (Coleoptera: Anobiidae) and overcoming control failures during fumigation of stored tobacco. Int J Pest Manag 40: 207-210.

Rajendran S, Narasimhan KS (1994b). The current status of phosphine fumigations in India, In: Highley E, Wright EJ, Banks HJ and Champ BR (eds). Proceedings of the 6th International Working Conference on Stored-Product Protection. CAB International: Canberra, Australia, pp 148-152.

Raymond M, Heckel DG, Scott JG (1989). Interactions between pesticide genes: model and experiment. Genetics 123: 543-552.

Ren YL, O'Brien IG, Whittle GP (1994). Studies on the effect of carbon dioxide in insect treatment with phosphine, In: Highley E, Wright EJ, Banks HJ and Champ BR (eds). Stored Products Protection. Proceeding of the 6th International Conference on Stored Product Protection. CAB, Wallingford, UK: Canberra, pp 173-177.

Saxena JD, Bhatia SK (1980). Laboratory selection of the red flour beetle, Tribolium castaneum (Herbst) for resistance to phosphine. Entomonograph 5: 301-306.

Schlipalius D, Collins PJ, Mau Y, Ebert PR (2006). New tools for management of phosphine resistance. Outlooks Pest Manag 17: 51-56.

Schlipalius DI (2004). Molecular Genetics and Linkage Analysis of Phosphine Resistance in the lesser grain borer, Rhyzopertha Dominica (Coleoptera: Bostrichidae). University of Queensland: Brisbane, Australia.

Schlipalius DI, Cheng Q, Reilly PE, Collins PJ, Ebert PR (2002). Genetic linkage analysis of the lesser grain borer Rhyzopertha dominica identifies two loci that confer high-level resistance to the fumigant phosphine. Genetics 161: 773-782.

Schlipalius DI, Waldron J, Carroll BJ, Collins PJ, Ebert PR (2001). A DNA fingerprinting procedure for ultra high-throughput genetic analysis of insects. Insect Mol Biol 10: 579-585.

Shono T, Kasai S, Kamiya E, Kono Y, Scott J (2002). Genetics and mechanisms of permethrin resistance in the YPER strain of house fly. Pestic Biochem Physiol 73: 27-36.

Tabashnik BE (1990). Modeling and evaluation of resistance management tactics, In: Roush RT and Tabashnik BE (eds). Pesticide Resistance in Arthropods. Chapman and Hall: New York and London, pp 153-182.

Tyler PS, Taylor RW, Rees DP (1983). Insect resistance to phosphine fumigation in food warehouses in Bangladesh. Int Pest Control 25: 10-13, 21.

White GG, Lambkin TA (1990). Baseline responses to phosphine and resistance status of stored-grain beetle pests in Queensland, Australia. J Econ Entomol 83: 1738-1744. 